\title{
Method of clad bead height evaluation based on analysis of laser impact area images
}

\author{
Darya Chkalova* \\ Vladimir State University, Gorky street, 87, Vladimir, 600000, Russia
}

\begin{abstract}
The work is devoted to solving the problem of height estimating of clad bead, formed by laser powder cladding technology, by analyzing the images of processing area. A technique for evaluating of deposited structures geometry is shown by comparing the sharpness differences of individual sections of studied images. An optical scheme is described that makes it possible to assess the depth of field of obtain images based on automated data analysis.
\end{abstract}

\section{Introduction}

Powder cladding technology is a thermal coating method widely used in industry to obtain wear-resistant and corrosion-resistant surfaces [1-3]. At present, the most promising approach of powder cladding, devoid of the disadvantages of other technologies, is the use of laser radiation as an energy source [4,5]. The interactive process between high power laser and metal material, leading to a high temperature and high pressure area, is a complex kinetics process with complicated physicochemical changes [6].

There are many factors that describe the quality of formed cladding, but the key parameter is the constancy of clad bead height. Thus, to control the process of powder laser cladding, first of all, it is necessary to implement the framework for registering the clad material height.

One of the ways to recognize the bead height, based on the principle of automated image analysis, is to estimate the sharpness depth of target parts of cladding area images the recorded by the camera. Since the bead is a volumetric object, the peak of the bead lies in a plane other than the plane of the substrate. Thus, based on the analysis of the sharpness depth of the clad area, it is possible to carry out an analytical measurement of the height difference to identify the volumetric geometry of the clad bead.

\section{Experiment scheme}

To obtain the geometric characteristics of the researched area, it is first of all necessary to determine the sharpness depth of the optical system. The clad bead cannot be entirely located in the zone of the registration system focus sharpness, since it has a certain height, width, length, radius, as a result of which the configuration of the optical system focal plane position should provide the possibility of obtaining the maximum amount of graphic information

\footnotetext{
* Corresponding author: j.larenax@gmail.com
} 
about the considered area geometry $[7,8]$. To solve the problem of studying the sharpness depth parameter, an experimental scheme was assembled, shown in Fig. 1.

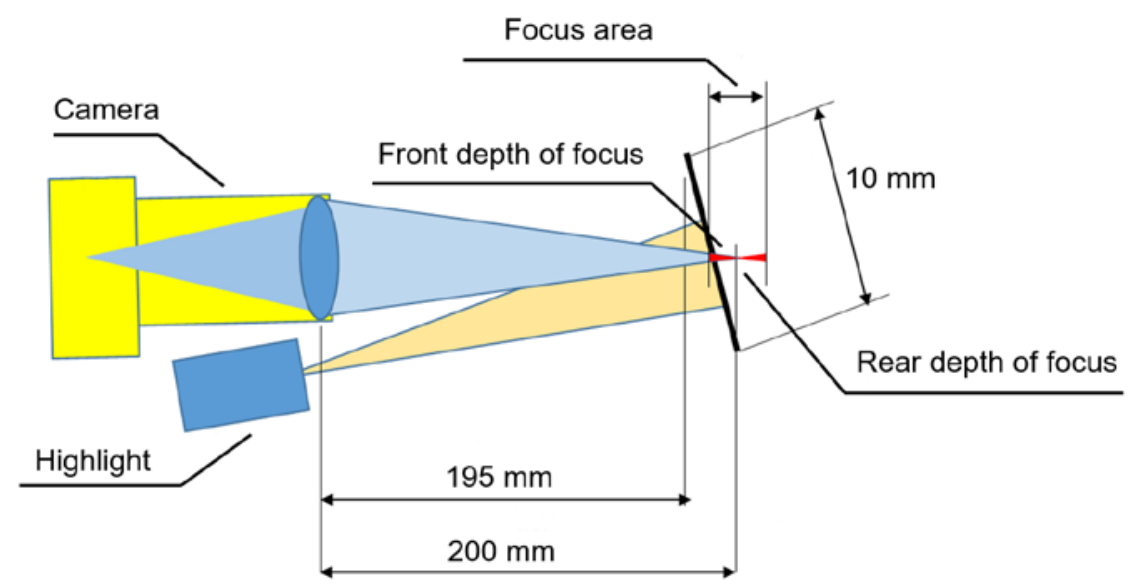

Fig. 1. The scheme for determining the sharpness depth of the photo-video fixation system.

The graduated plate was placed at an angle of $30^{\circ}$ to the plane of the photo and video fixation system. One side of the scale was located in the focal plane of the optical system at a distance of $200 \mathrm{~mm}$, the other side was located at a distance of $195 \mathrm{~mm}$. The image was blurred, but recognizable at a distance of $195 \mathrm{~mm}$, allowing identification of the considered object (Fig. 2).

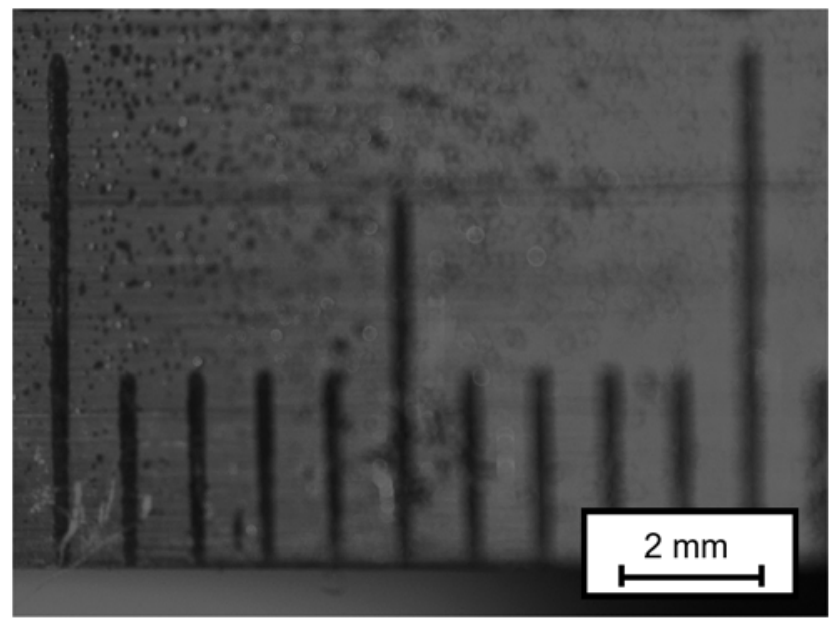

Fig. 2. Image of a graduated scale located at an angle of $30^{\circ}$ to the camera plane, graduated scale divisions is $1 \mathrm{~mm}$.

By visual assessment, it was identified that the high contrast of the image is over an area of $2 \mathrm{~mm}$ in length (on the plate scale). Taking into account the angle of graduated plate inclination, the value of the focus area for the considered case is about $1 \mathrm{~mm}$. Thus, based on analysis of sharpness depth of considered area two-dimensional images, it is possible to carry out an analytical measurement of height difference to identify dimensional characteristics of objects, such as height of the clad bead. 


\section{Image sharpness estimation method}

To automate the process of determining focus sharpness zone (for purpose of further calculating the bead height), it is necessary to carry out a mathematical assessment of blurring degree of image areas. The image evaluation process includes several stages, the most important of which is selection of image elements boundaries. The problem of selecting the boundaries was solved by overlaying simple masks on images, the weight coefficients of which can take both positive and negative values. The principle of such processing is as follows: the pixel of new image is formed on the basis of original image pixel, taking into account the various weight coefficients of applied mask (Fig. 3).

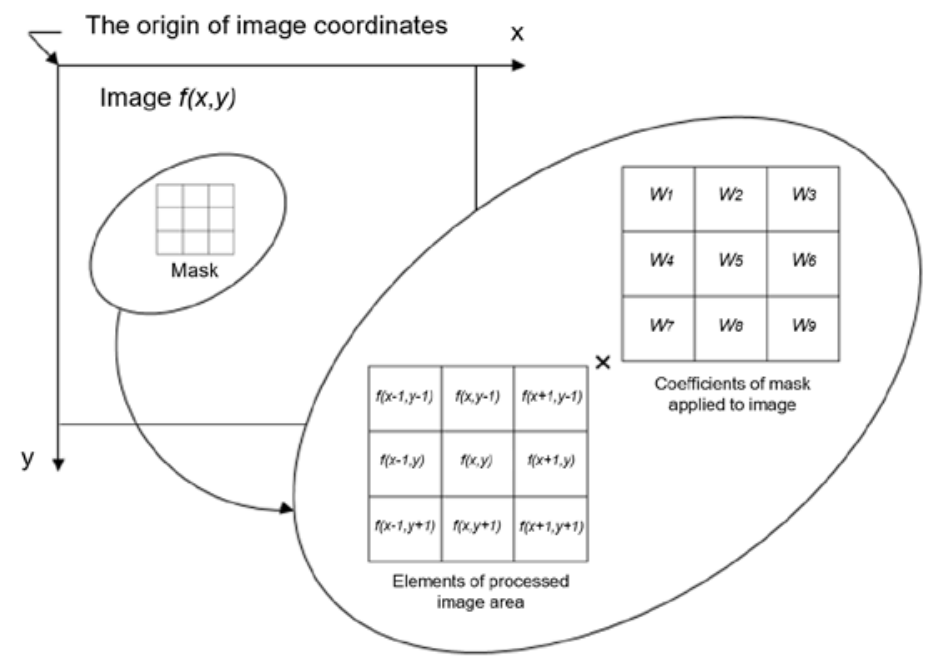

Fig. 3. Image spatial filtering scheme.

The pixels have approximately the same brightness in monochrome areas of the image, the gradient of which is low, and in the resulting image, such areas darken. In areas of sharp changes in brightness, characterized by contours of images elements, the steepness of the brightness change is high, and in the final image white contour lines appear in such areas.

One of the most widely used contour extracting filters is the Laplace filter [9,10]. As a result of converting the original image into achromatic with its further processing using this filter, the distribution of pixel brightness will lie in the range from 0 (black color, the greatest blur) to 255 (the most contrasting areas of the original image). The diagram of the distribution of the brightness of the pixels of Fig. 2 processed by the Laplace filter is shown in Fig. 4.

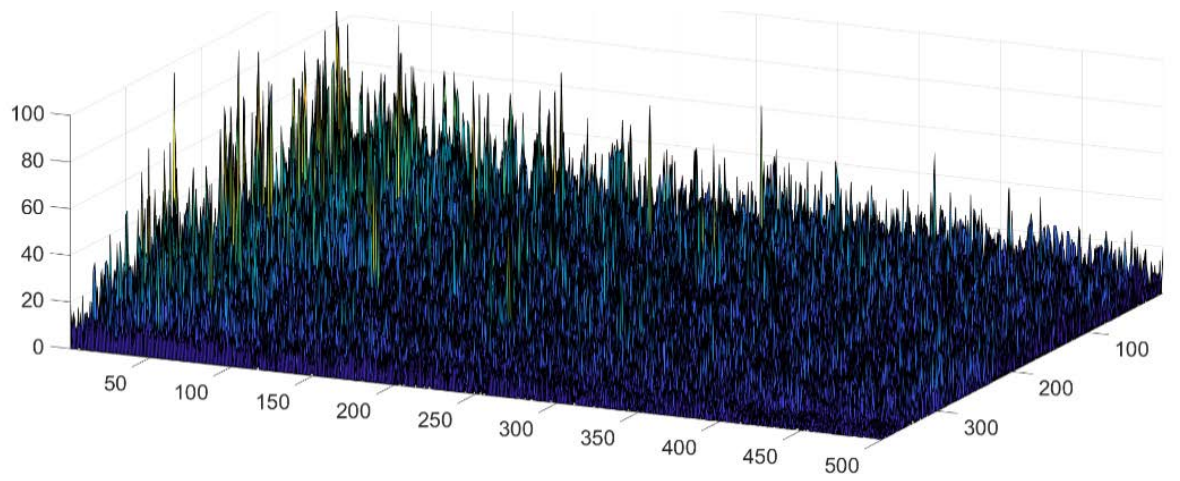

Fig. 4. The diagram of pixels brightness distribution of the processed image. 
As can be seen from the diagram, the higher the peaks, the sharper the corresponding area of the original image is. In this case, average value of the square of the pixel brightness should be taken as a parameter allowing to compare the degree of blurring of several areas for the image:

$$
\bar{Y}^{2}=\frac{1}{N} \sum_{p=1}^{N} Y_{p}{ }^{2},
$$

where $Y_{p}$ - the brightness of each pixel in the image; $N$ - number of pixels in estimated area. The smallest degree of blur will correspond to the image area with the largest value $\bar{Y}^{2}$, which means that it belongs to the sharpness zone of the optical system. Pixels with a brightness of less than 10 units (the working range is $0-255$ ) were excluded from the calculation, since they are not informative.

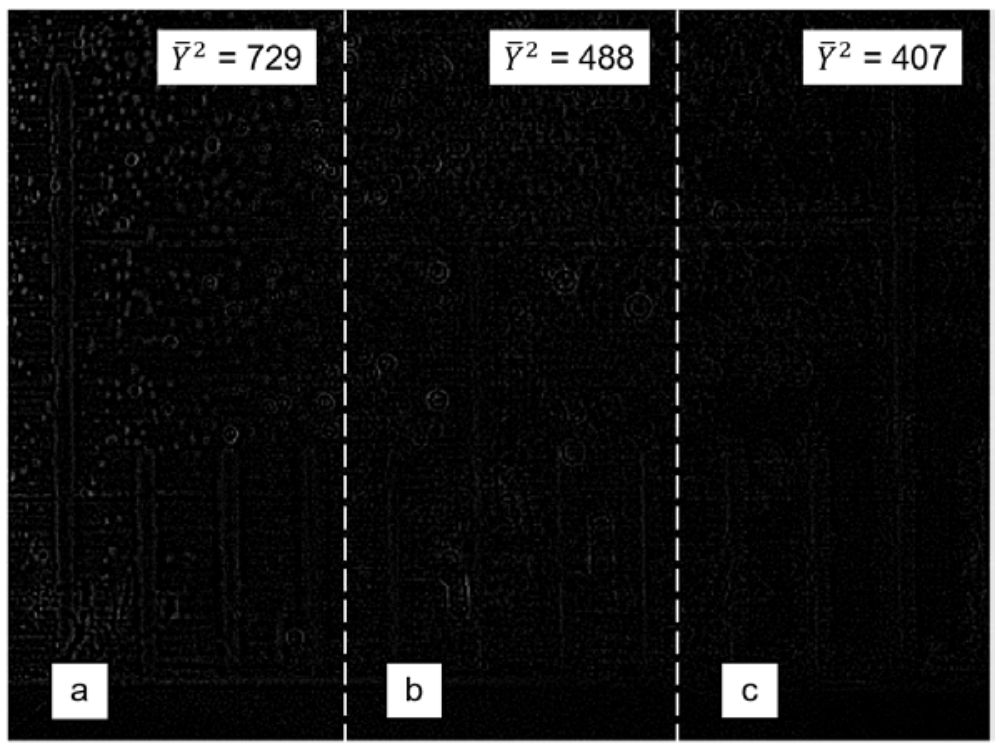

Fig. 5. The processing result of image 2 by Laplace filter.

According to the calculated data presented in Fig. 5, we can conclude that the region "a" of the original image is much more contrasting for the algorithm than "b" and "c" (it corresponds to the results of visual assessment), thereby guaranteeing its entry into the zone sharpness of the optical system.

\section{Conclusion}

Thus, after a calculation of the video recording system optical parameters on the basis of the above algorithm, it becomes possible to implement an automated analytical assessment of the clad beads height. The control of geometric characteristics by evaluating the sharpness of image is an effective method, provided that a preliminary calibration of the system is performed to determine the exit vector of processing plane from the focus area. 


\section{References}

1. R.S. Korsmik, G.A. Turichin, O.G. Klimova-Korsmik, E.V. Alekseeva, R.S. Novikov, Development of laser powder cladding technology for restoration of heat-resistant nickel alloys turbine blades, J. of Phys., v. 1109, pp. 12-23 (2018)

2. J. Y. Jeng, S.C. Peng, C.J. Chou, Metal rapid prototype fabrication using selective laser cladding technology, The International Journal of Advanced Manufacturing Technology, v. 16(9), pp. 681-687 (2000)

3. Q. Lai, R. Abrahams, W. Yan, C. Qiu, P. Mutton, A. Paradowska, M. Soodi, Investigation of a novel functionally graded material for the repair of premium hypereutectoid rails using laser cladding technology, Composites Part B: Engineering, v. 130, pp. 174-191 (2017)

4. R. Huang, J. Luo, C. Wang, Applications and developments of laser cladding technology, Ordnance Material Science and Engineering, v. 4 (2005)

5. Q. Liu, M. Janardhana, B. Hinton, M. Brandt, K. Sharp, K., Laser cladding as a potential repair technology for damaged aircraft components, International Journal of Structural Integrity, v. 2(3) (2011)

6. J. Yang, H. Ouyang, Y. Wang, Direct metal laser fabrication: machine development and experimental work, The International Journal of Advanced Manufacturing Technology, v. 46, pp. 1133-1143 (2010)

7. T. Kannan, J. Yoganandh, Effect of process parameters on clad bead geometry and its shape relationships of stainless steel claddings deposited by GMAW, The International Journal of Advanced Manufacturing Technology, v. 47, pp. 1083-1095 (2010)

8. S. Saqib, R.J. Urbanic, K. Aggarwal, Analysis of laser cladding bead morphology for developing additive manufacturing travel paths, Procedia Cirp., v. 17, pp. 824-829 (2014)

9. M. Aubry, S. Paris, S.W. Hasinoff, J. Kautz, F. Durand, Fast local Laplacian filters: Theory and applications, ACM Transactions on Graphics (TOG), v. 33(5), pp. 1-14 (2014)

10. S. Paris, S., S.W. Hasinoff, J. Kautz, Local Laplacian filters: Edge-aware image processing with a Laplacian pyramid, ACM Trans. Graph., v. 30(4), p. 68 (2011) 